Universidad de Lima

Facultad de Ciencias Empresariales y Económicas

Carrera de Economía

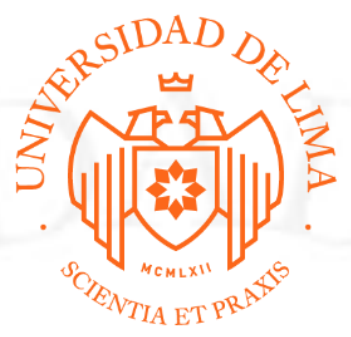

\title{
DESCUBRIENDO EL MERCADO DE CAPACITACIÓN PARA MYPE: UN ANÁLISIS DE LA OFERTA, DEMANDA Y EFICACIA DE LOS PROGRAMAS DE CAPACITACIÓN
}

Trabajo de investigación para optar el Título Profesional de Economista

\section{Walter Miguel Cuba Rojas}

Código 20110357

\author{
Asesor \\ Yuri Jesus Landa Arroyo
}

Lima - Perú

Abril de 2019 


\section{DESCUBRIENDO EL MERCADO DE CAPACITACIÓN PARA MYPE: UN ANÁLISIS DE LA OFERTA, DEMANDA Y EFICACIA DE LOS PROGRAMAS DE CAPACITACIÓN}




\section{TABLA DE CONTENIDO}

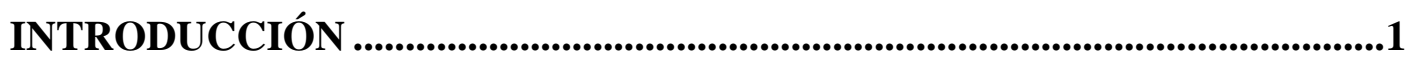

CAPÍTULO I: SITUACIÓN DE LAS MYPE.........................................................4

1.1. Características generales ...................................................................

1.2. Evolución empresarial .........................................................................6

1.3. Hitos en el mercado de capacitación ............................................................

CAPÍTULO II: MARCO TEÓRICO ....................................................................9

2.1. Demanda y oferta de capacitación ............................................................ 10

2.2. Informalidad y capacitación microempresarial ............................................... 11

2.3. Efectividad de los programas de capacitación .............................................12

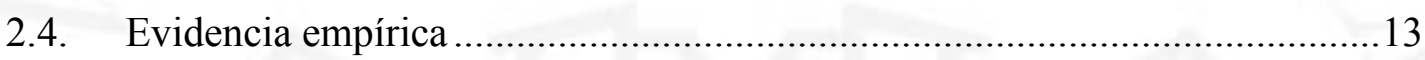

CAPÍTULO III: EVALUACIÓN EMPÍRICA …............................................................16

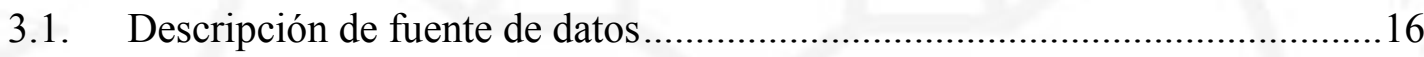

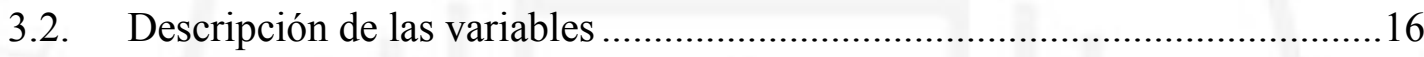

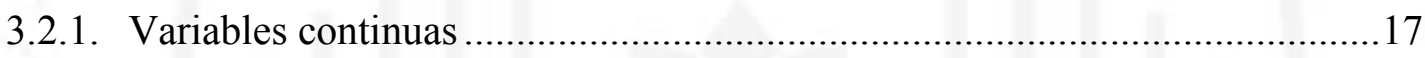

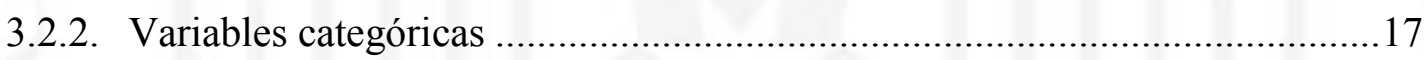

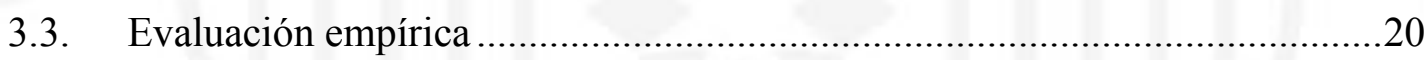

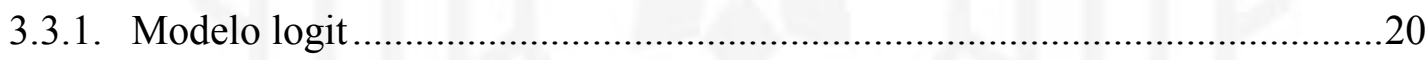

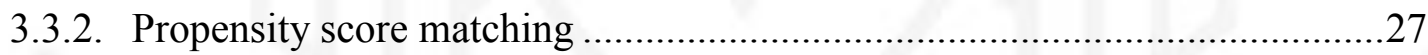

CAPÍTULO IV: ORGANIZACIÓN DE LAS CAPACITACIONES ...................30

CAPÍTULO V: CONCLUSIONES Y RECOMENDACIONES .........................34

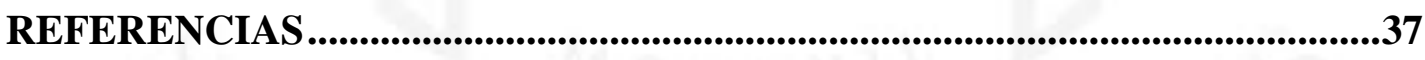

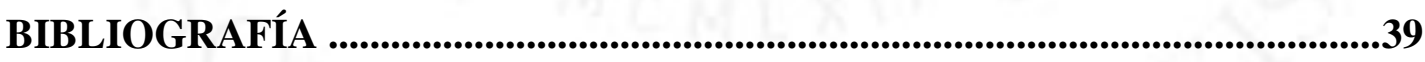




\section{ÍNDICE DE TABLAS}

Tabla 1.1 Matriz de transición empresarial .............................................................6

Tabla 3.1 Número de observaciones por encuesta EMYPE ........................................ 16

Tabla 3.2 Estadísticos de las variables edad, utilidad operativa, antigüedad y

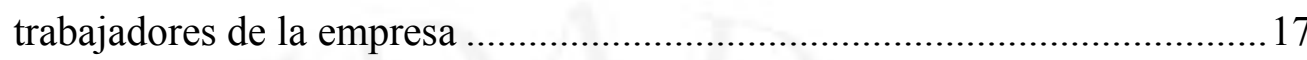

Tabla 3.3 Frecuencias de las variables capacitación empresarial, género, licencia municipal, cantidad de equipos y actividad empresarial .............................. 18

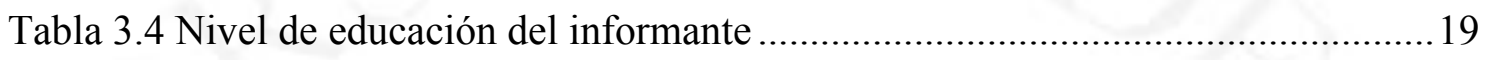

Tabla 3.5 Provincia de la empresa encuestada ..........................................................20

Tabla 3.6 Resultados del primer modelo econométrico .............................................22

Tabla 3.7 Estimación del efecto de la capacitación sobre las utilidades operativas ........29 


\section{ÍNDICE DE FIGURAS}

Figura 1.1 Ventas anuales durante el 2013 en miles de soles......................................

Figura 1.2 Distribución de las MYPE según actividad económica .................................5

Figura 1.3 Tasa de entrada bruta - Tasa de salida bruta .............................................. 6

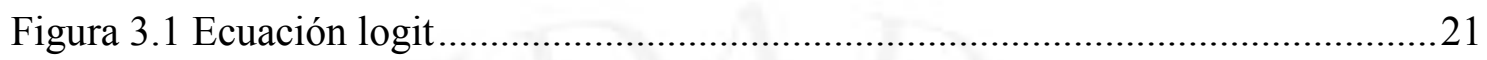

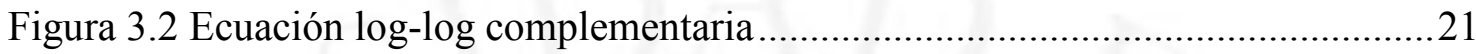

Figura 3.3 Porcentaje de observaciones correctamente calificadas según el modelo.....24

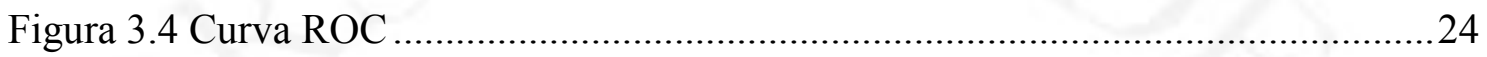

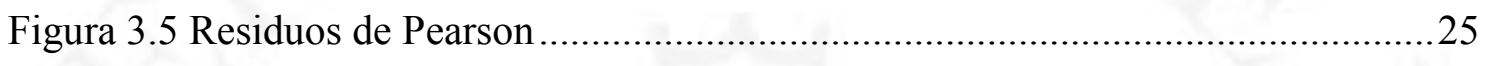

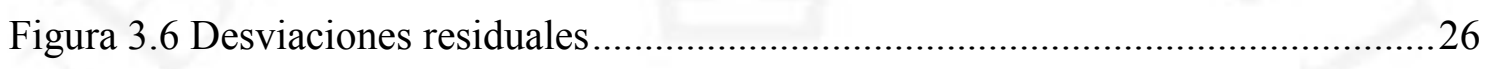

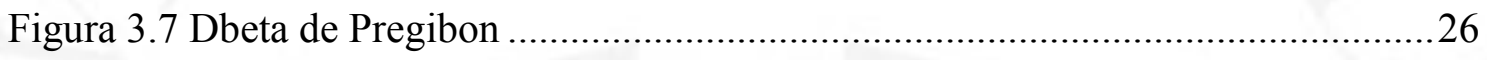

Figura 3.8 Utilidad operativa antes del tratamiento de datos atípicos ..........................28

Figura 3.9 Utilidad operativa luego del tratamiento de datos atípicos ..........................28 


\section{INTRODUCCIÓN}

Las Micro y Pequeñas Empresas (MYPE) tienen un peso importante en la economía peruana, según cifras del Ministerio de la Producción (PRODUCE, 2017) las MYPE concentran el 58,6\% de la Población Económicamente Activa (PEA) ocupada y el 23,5\% del valor agregado del sector privado. Estas empresas enfrentan diversos retos en su constitución y desarrollo posterior, entre ellos se encuentra el financiamiento de sus operaciones, la baja productividad y la informalidad (de acuerdo a cifras de PRODUCE (2017) el número de microempresas aumenta de 1,6 millones a 3,5 millones si se toman en cuenta las empresas informales). Por ello, es relevante realizar un estudio acerca de uno de los factores que pueden llevar a una MYPE a ser exitosa: la capacitación gerencial.

El mercado de capacitación para las MYPE ha sido estudiado parcialmente para el caso peruano, pues los estudios no abordan el tema en su cabalidad y se limitan a ejecutar análisis cualitativos a pesar de que, según la opinión de los expertos, una de las principales carencias de los emprendimientos actuales es la falta de una gerencia adecuada en la empresa. Por ello, es necesario realizar un análisis del tema con mayor rigurosidad.

Es importante mencionar que las políticas de apoyo para la creación de las MYPE deben tener siempre un componente de promoción del desarrollo de estas empresas, pues existe el riesgo de entorpecer el crecimiento debido a los incentivos perversos que existen por los beneficios que el estado suele ofrecer (Agosin et al., 2010). Actualmente, en el Perú la proporción de micro y pequeña empresa que no logran crecer y subir de categoría es bastante alta, los porcentajes fueron de $92 \%$ y $60 \%$ respectivamente para el periodo del 2008 al 2015 (PRODUCE, 2017). Por otro lado, se encuentra que en los países en desarrollo las fuerzas del mercado no son suficientes para incentivar a las empresas a mejorar continuamente (Bloom, Mahajan, McKenzie, \& Roberts, 2010).

En el mercado de capacitación MYPE (en adelante mercado de capacitación microempresarial) la oferta está compuesta principalmente por: el Ministerio de Producción, el Ministerio de Trabajo y Promoción del Empleo (MINTRA), Municipalidades Distritales y COFIDE, luego de estas entidades se encuentran diferentes organizaciones privadas (entidades financieras, universidades, organismos no gubernamentales, entre otros). Según Matute, Albújar, Janampa, Odar y Osorio (2008) 
existe una falta de coherencia entre lo que se enseña y lo demandado por los microempresarios, asimismo, se señala que el MINTRA no tiene la capacidad para concertar esfuerzos entre los diferentes proveedores de capacitación microempresarial.

El estudio de Matute et al. (2008) señala que el 96\% de los empresarios encuestados consideraba que recibir capacitación era importante, sin embargo, de acuerdo a cifras del Instituto Nacional de Estadística e Informática (INEI) calculadas a partir de la Encuesta de Micro y Pequeña Empresa, el porcentaje de microempresarios que llega a capacitarse es aproximadamente de $30 \%$ por año. Esta paradoja motiva el estudio y análisis de la demanda de capacitación.

Los objetivos de la investigación son:

Objetivo General:

Analizar el mercado de capacitación microempresarial, en cuanto a la interacción de la oferta y demanda y resultados de la transacción, en el horizonte temporal del 20102013 teniendo el estudio representatividad a nivel de ciudades grandes.

Objetivos específicos:

Determinar las características de los empresarios que generan incentivos para capacitarse y señalar aquellas que pueden afectar la efectividad de los programas de capacitación si no son tomados en cuenta por la oferta.

Verificar la efectividad de los programas de capacitación en cuanto al aumento de los ingresos percibidos por quienes recibieron la capacitación.

Asimismo, se plantean las siguientes hipótesis acerca del mercado de capacitación empresarial:

Hipótesis General:

Existen diferencias entre las características de la empresa y el empresario que suele capacitarse que podría generar problemas de selección en los programas de capacitación, los cuales tienen efectos positivos en los ingresos percibidos en el negocio.

Hipótesis Específicas:

Existen factores que influyen en la decisión de capacitarse que vienen tanto de las características de la empresa como del dueño, siendo estos últimos los más importantes. 
Los programas de capacitación microempresarial aumentan los ingresos de las empresas en el corto plazo debido a las mejores prácticas que adoptan los receptores de la capacitación.

Para comprobar las hipótesis se utilizará la base de datos "Encuesta de Micro y Pequeña Empresa (EMYPE)" para los años del 2010 al 2013. Esta fuente de información es elaborada por el INEI y tiene representatividad a nivel de ciudades grandes las cuales son elegidas por el INEI debido a su relevancia económica, el número de observaciones por cada año es aproximadamente 3,000.

La investigación realizada se distingue de las hechas previamente por su carácter cuantitativo y la caracterización de una demanda de capacitación, además por su enfoque en la problemática de las MYPE.

Para la primera hipótesis específica se plantea el uso de un modelo de variable dependiente limitada para ver qué variables aportan más a la probabilidad de que un empresario haya sido capacitado. Este modelo econométrico recibe tal nombre dado que la variable a estudiar solo varía entre valores acotados (en nuestro caso se puede obtener dos resultados: haber sido capacitado o no). Es importante mencionar que estos modelos son más conocidos como logit o probit, dependiendo de la distribución asumida para los valores modelados.

Respecto a la segunda hipótesis específica se realizará una evaluación de impacto mediante el propensity score matching (PSM) para ver si las utilidades operativas se relacionan con el haber sido capacitado. Para ello serán necesarios los resultados del primer modelo econométrico

El trabajo está organizado de la siguiente manera. El primer capítulo abarca la situación de las MYPE en torno al tema de capacitación empresarial, el segundo capítulo es el marco teórico del trabajo en el cual se mostrará teoría y evidencia empírica acerca de la demanda de capacitación y resultados de la capacitación empresarial. En el tercer capítulo se realizará un modelo PSM para identificar el efecto que tiene la capacitación sobre los ingresos. El cuarto capítulo contiene una descripción de las capacitaciones empresariales y su organización, también se incluye observaciones hechas sobre el desarrollo de las mismas. Por último, el quinto capítulo son las conclusiones y las recomendaciones del estudio. 


\section{CAPÍTULO I: SITUACIÓN DE LAS MYPE}

\subsection{Características generales}

Entender el tema de la capacitación de los empresarios de las MYPE supone entender primero como está organizado este sector y cuáles son los retos que enfrenta. De acuerdo a la Ley MYPE (Ley $N^{\circ} 30056,2013$ ), una empresa es micro si sus ventas anuales son menores a 150 Unidades Impositivas Tributarias (UIT) y es pequeña si son mayores que 150 UIT pero menores que 1700 UIT. El sector de las MYPE, según datos de PRODUCE (2017), comprende el 99\% de las empresas formales, lo que equivale a 1,691,462 empresas, mientras que las microempresas informales son 1,852,351.

La mayoría de las empresas factura muy poco en ventas anuales. Se puede constatar que el 50\% de las MYPE consiguen vender menos de 5 UIT al año y casi el $40 \%$ menos de 2 UIT al año (la UIT del 2015 se encontraba en S/ 3,850).

Figura 1.1

Ventas anuales durante el 2013 en miles de soles

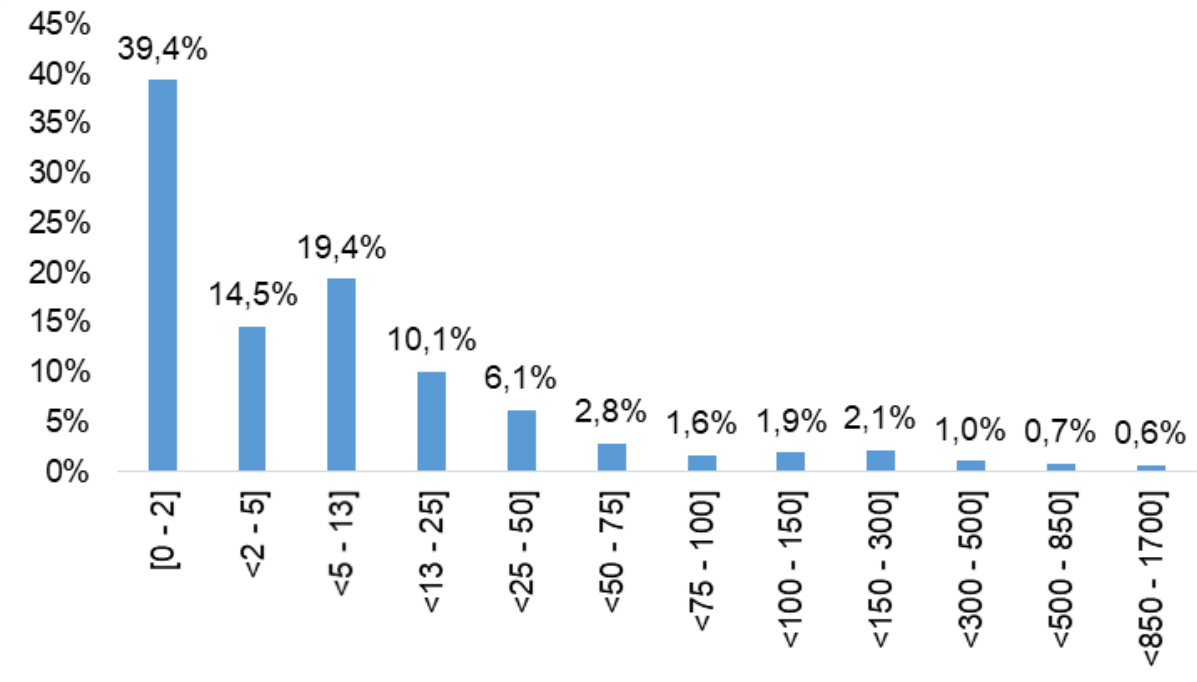

Fuente: PRODUCE (2017)

Por otro lado, Lima tiene la mayor participación de MYPE formales con un 48\%, seguido de Arequipa con 6\% y La Libertad con 5\%. Asimismo, para los sectores en los que operan las MYPE se puede ver otra gran asimetría, pues la mayoría de empresas se 
concentra en el sector comercio y servicios. La misma proporción presentada se ha mantenido desde el 2009 (PRODUCE, 2017).

Figura 1.2

Distribución de las MYPE según actividad económica

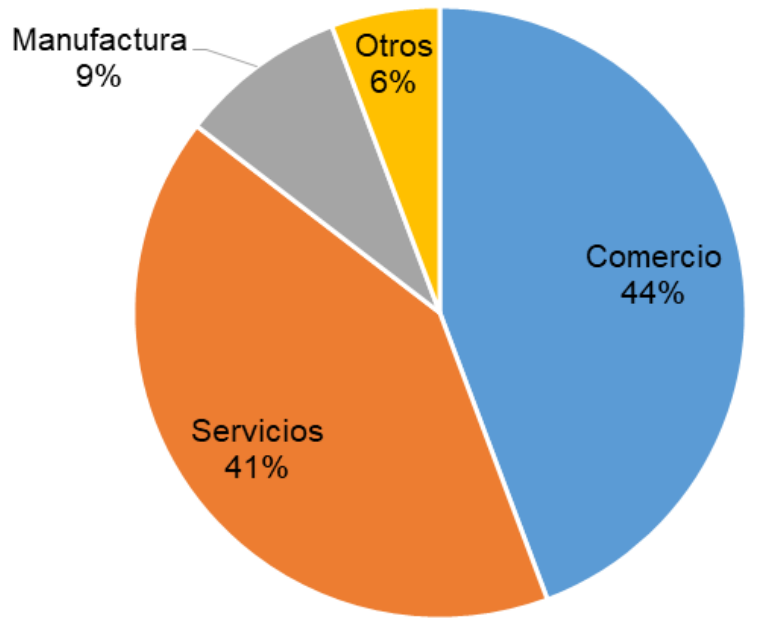

Fuente: PRODUCE (2017)

Adicionalmente, según cifras de PRODUCE (2017), el 59\% de las microempresas tuvieron 5 años de tiempo de vida en el mercado durante el 2013, el porcentaje fue de $45,2 \%$ para la pequeña empresa. Por otro lado, según Villarán (2010), cerca de la tercera parte de los trabajadores de las microempresas son familiares no remunerados y el $94 \%$ no accede a beneficios sociales. El hecho de tener muchas empresas manejadas por familias incide en la productividad de los trabajadores negativamente, pues las probabilidades de ser despedido a causa de no rendir adecuadamente no son altas.

Es importante señalar que la tasa de entrada (empresas creadas en un periodo respecto al total de empresas existentes) y la tasa de salida (empresas que cierran en un periodo respecto al total de empresas existentes) de las microempresas son las mayores entre las demás categorías de empresas. 
Figura 1.3

Tasa de entrada bruta - Tasa de salida bruta

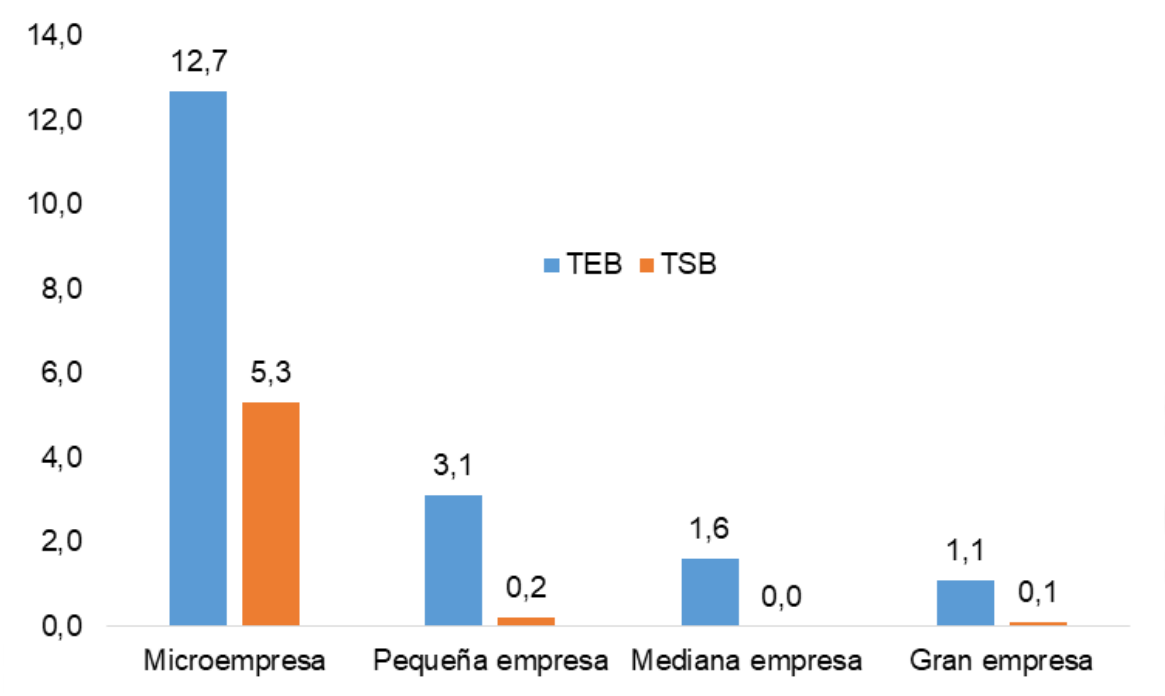

Fuente: PRODUCE (2017)

\subsection{Evolución empresarial}

Acerca de la transición de las MYPE a categorías superiores, PRODUCE construye la siguiente matriz de transición para describir la dinámica empresarial.

Tabla 1.1

Matriz de transición empresarial

\begin{tabular}{|l|c|c|c|c|}
\hline $\begin{array}{l}2015 \\
2008\end{array}$ & Microempresa & Pequeña & Mediana & Grande \\
\hline Microempresa & $\mathbf{9 2 , 2}$ & 7,3 & 0,2 & 0,4 \\
\hline Pequeña & $\mathbf{3 7 , 8}$ & $\mathbf{5 3 , 5}$ & 2,9 & 5,8 \\
\hline Mediana & 16,7 & 30,3 & 3,0 & 50,0 \\
\hline Grande & 20,0 & 13,8 & 3,8 & 62,3 \\
\hline
\end{tabular}

Fuente: PRODUCE (2017)

Se puede observar que el $92,2 \%$ y $53,5 \%$ de las microempresas y pequeñas empresas, respectivamente, mantuvieron la clasificación que tenían en el 2008. Por otro lado, se puede ver que el $37,8 \%$ de las empresas que eran consideradas pequeñas en el 2008 fueron clasificadas como microempresas en el 2013.

Las probabilidades de que una empresa crezca son muy pequeñas. Esto se puede explicar por dos motivos: Factores limitantes en el negocio o pocos motivantes de desarrollo. Tradicionalmente se han hablado de dos motivos para la creación de una 
empresa: el de necesidad, que surge cuando una persona queda desempleada o ve que los ingresos corrientes no le son suficiente; y, el motivo de oportunidad, que aparece cuando una persona busca emprender un nuevo negocio con aspiraciones a desarrollarse dado el buen ambiente económico y las oportunidades que percibe. Al respecto, el Global Entrepreneurship Monitor (GEM) realiza encuestas para ver qué porcentaje de los emprendedores son guiados por el motivo oportunidad y por el motivo necesidad. En los últimos años (desde el 2010 al 2017) se ha observado que el motivo de necesidad explica aproximadamente el 90\% de los emprendimientos del país.

Un factor limitante del crecimiento es la alta relación con la informalidad de las MYPE. Al no estar dentro de la economía formal, las empresas no tienen la capacidad de crecer por temas de licencia y por no tener acceso al crédito formal. Estimaciones de PRODUCE usando la Encuesta Nacional de Hogares (ENAHO) muestran que el número de MYPE informales llegaría a ser de 3,060,461, un poco más del doble de empresas formales (PRODUCE, 2017).

\subsection{Hitos en el mercado de capacitación}

El tema de la capacitación de los microempresarios ha tenido algunos hitos importantes, los cuales fueron la ley 28015 en el 2003, el decreto legislativo 1086 en el 2008, el decreto supremo 024-2009 y la ley 30056 en el 2013.

En la ley 28015, también llamada Ley MYPE, se dedican los artículos 16, 17 y 18 a la capacitación y asistencia técnica de las MYPE. Aquí se especifica que el estado promueve estos programas a través de Consejo Nacional para el Desarrollo de la Micro y Pequeña Empresa (CODEMYPE), la prioridad de temas de capacitación son:

"La creación de empresas, la organización y asociatividad empresarial, la gestión empresarial, la producción y productividad, la comercialización y mercadotecnia, el financiamiento, las actividades económicas estratégicas y los aspectos legales y tributarios." (Ley No 28015, 2003)

También, se especifica que se apoya la iniciativa privada y que el Servicio Nacional de Adiestramiento del Trabajo Industrial (SENATI) también ayudará en estos temas. Asimismo, menciona que el Ministerio de Trabajo y Promoción del Empleo en coordinación con el Ministerio de Educación se encargan de reconocer a las instituciones 
que brindan capacitación, como entidades educativas. Se tiene así un marco regulatorio para la capacitación de MYPE.

En el decreto supremo 029-2009 de PRODUCE se estipula que las microempresas sujetas al régimen especial laboral podrán permanecer de esa forma hasta el 4 de julio del 2013, luego estas empresas pasarían a estar en el régimen laboral general. Luego, en el decreto legislativo 1086 se establecería un tipo de régimen laboral especial que a diferencia del otro sería permanente. Otro punto importante del decreto legislativo 1086 es que se menciona algunos instrumentos de promoción para las microempresas, como el establecimiento del Fondo de Investigación y Desarrollo de la Competitividad (FIDECOM) que financiaría a los programas de capacitación y a los microempresarios que busquen innovar en su negocio. También se menciona que el acceso a los programas se priorizará en las empresas que estén asociadas con otras.

Por último, la ley 30056 establece que la clasificación de las empresas será solo de acuerdo a su nivel de ventas, esto permitiría a las MYPE contratar a más personas sin tener que perder su condición de micro o pequeña empresa. También, se ratifica el apoyo del estado a las iniciativas privadas de capacitación. Se habla de que el estado apoya el crecimiento de las empresas mediante programas de capacitación en calidad, de forma que se cumplan con estándares internacionales. Cabe decir, que en esta ley la prórroga del tiempo de vigencia del régimen especial establecido en la ley 28015 se extiende por tres años más. 


\section{CAPÍTULO II: MARCO TEÓRICO}

La decisión de capacitarse parte de un análisis de costo-beneficio realizado por el microempresario, se sopesa cual es el costo de capacitarse (monetario o no monetario) y los beneficios de hacerlo, los cuales tradicionalmente se refieren a una reducción de los costos de la empresa al gestionarla de una manera más eficiente (Becker, 1962). Por lo tanto, la capacitación se asemeja a una decisión de inversión, pues hay un periodo inicial en donde se emplea tiempo o se gasta dinero para entrenarse y en los siguientes periodos se espera que este aprendizaje dé ganancias (costos ciertos y beneficios inciertos).

Una empresa al adquirir capacitación rentable e implementar las mejores prácticas en la gestión aprendidas, tendrá una ventaja competitiva sobre las demás empresas. Esta empresa capacitada podría atraer a los clientes de las empresas mal gestionadas al ofrecer un servicio mejor o aprovechar sus ventajas en costos (Walker, 1887) menciona que las empresas exitosamente gestionadas son capaces de reducir sus gastos), de esta forma, las empresas mal gestionadas terminarían desapareciendo, sin embargo, no es lo que se observa en los datos. Bloom et al. (2010) encuentran una persistencia de empresas mal manejadas en países en desarrollo, los autores explican el fenómeno señalando la falta de competencia, es decir, las empresas bien gestionadas y mal gestionadas compiten en mercados diferentes. Esto se podría aplicar en cierta medida para el Perú, en el sentido que existen diferentes tipos de consumidores (nivel socioeconómico, hábitos de consumidor, entre otras características) que hacen que los mercados se segmenten y no compitan entre ellos.

Los microempresarios muchas veces son los dueños y trabajadores de la empresa. El microempresario tiene que enfrentarse a la decisión entre cuanto permitirse descansar, trabajar y cuánto de los resultados de la empresa utilizar para el consumo o inversión. Es por ello que en la literatura que se menciona a continuación se aborda el tema desde ambos puntos de vista, de un empresario que paga por la capacitación de sus trabajadores y de un trabajador que quiere o acepta capacitarse. 


\subsection{Demanda y oferta de capacitación}

Una primera dificultad al momento de desarrollar un marco teórico acerca de la capacitación microempresarial, es que solo se encuentran trabajos para grandes empresas. En este sentido, es necesario la adecuación de la teoría al caso específico estudiado.

Entre los primeros autores que se refieren a este tema se encuentra a Becker (1962). Él nos habla de dos tipos de capacitación: general y específica. La primera es aquella que eleva la productividad de un trabajador independientemente del rubro o giro de la empresa en la cual trabaje, en cambio la capacitación específica eleva la productividad solo en la empresa para la cual ha sido diseñada la capacitación, esto es debido a que el segundo tipo de capacitación solo ofrece mejorar las habilidades en un ámbito específico, por ejemplo, ensamble de computadoras, mientras que el primer tipo de capacitación se refiere a temas generales como administración de empresas. El autor argumenta que los incentivos que tiene una empresa para capacitar a un trabajador en forma general se generan por la expectativa de una mayor productividad. Sin embargo, los beneficios de tener personal más capacitado se anulan por el mayor salario que se les tendría que pagar. Por ello, el único incentivado para pagar por la capacitación es el mismo empleado, pues desea recibir un salario mayor en el futuro en la empresa donde trabaja o en otra. En el caso de una capacitación específica, la empresa es la única que va a poder beneficiarse de los resultados, por lo que sí tiene un incentivo para pagar la capacitación a sus trabajadores. Asimismo, deberá aumentar el salario para evitar el riesgo a que algún empleado capacitado renuncie, pues la empresa no quiere arriesgarse a perder su inversión. Se puede ver que los principales incentivos de las capacitaciones para la empresa y la persona son la mayor productividad y los mayores salarios, respectivamente.

El modelo que desarrolla Becker (1962) se concibe en un escenario de competencia perfecta en el mercado laboral, lo cual es improbable que se cumpla en la mayoría de los casos, sobre todo en un país en desarrollo. Acemoglu y Pischke (1999) argumentan que, cuando se levanta el supuesto de competencia perfecta, aún en el caso de capacitación general existen incentivos de la empresa a pagar por ella debido a que en equilibrio se paga menos que la productividad, pues debido al poder que tiene la empresa (poder monopsónico), se paga un salario menor a la productividad del personal. La existencia de este diferencial brinda un margen de ganancia que permite solventar los 
costos de la capacitación. Otra característica de este modelo es que el crecimiento de la productividad ante una hora adicional de capacitación es mayor que el crecimiento del salario, es decir, el grado de concavidad de la función de la productividad es mayor que el grado de la función de los salarios. Estas dos características generan los incentivos para que la empresa decida pagar por la capacitación existiendo un punto donde el costo marginal iguala al beneficio marginal.

Respecto a la oferta de capacitación, no existe literatura específica para el tema en cuestión, sin embargo, la teoría de los bienes públicos e información asimétrica nos pueden dar algunas luces acerca del problema. La capacitación es un bien público en el ámbito geográfico donde se está otorgando pues no existe rivalidad ni exclusión (salvo que se exceda la capacidad de aforo que se tenga en el local donde se esté capacitando). Como es bien sabido, los demandantes no tienen los incentivos a revelar la valoración del servicio que se les brinda, por lo que existen problemas de sub-oferta.

Por otro lado, la información asimétrica (cuando en una transacción una de las partes no puede tener acceso a información relevante que la otra parte no tiene incentivos a revelar) toma parte cuando se analizan los problemas de agencia que pueden existir. Si los sistemas de monitoreo son ineficientes, se puede dar el caso en el que el encargado del diseño de la capacitación no se esfuerce lo suficiente en realizar estudios para ver las necesidades de la demanda y decida ofrecer capacitaciones genéricas sin sustento técnico de modo de maximizar su propia utilidad.

\subsection{Informalidad y capacitación microempresarial}

Dado que la informalidad es una característica de suma relevancia en el sector de las MYPE, es importante mencionar la relación que tiene con la capacitación microempresarial.

Al respecto, La Porta y Shleifer (2014) señala que las empresas informales tienden a ser menos capacitadas, por lo tanto, es frecuente encontrar empresas de este tipo con un nivel muy bajo de productividad. Si bien el autor no da una explicación directa de por qué las personas informales no eligen capacitarse, el vínculo faltante puede estar en los bajos retornos que obtienen, pues están obligados a estar más horas en el trabajo para conseguir un mismo resultado. Asimismo, La Porta y Shleifer (2014) encuentra que la mayoría de empresas pequeñas (que el autor asocia con la informalidad) no tienen deseos 
de crecer, lo cual recuerda a los motivantes para iniciar una MYPE discutidos líneas arriba.

De forma similar Loaysa (2008) menciona que conforme un país tenga mayores niveles de educación la informalidad debería disminuir, pues la productividad laboral es mayor. Por lo tanto, el estar más capacitado puede explicar un cambio de informal a formal, sin embargo, el ser informal explica a la capacitación en la medida que es indicador de otros aspectos como baja productividad o escasos deseos de seguir creciendo.

\subsection{Efectividad de los programas de capacitación}

El primero en analizar el éxito de un negocio a causa de la gestión de la empresa es Walker (1887) quien observa que algunas empresas tienen más éxito que otras a pesar de tener el mismo nivel de capital, estar en la misma industria y tener las mismas oportunidades. Esto lo explica por las diferencias en el grado de habilidad de los empresarios para dirigir un negocio, pues al manejar eficientemente la empresa se prevén emergencias y contingencias, se minimiza el desperdicio, se satisface la demanda a tiempo y se logran condiciones favorables de crédito.

Por otro lado, Marshall (1890) refiere que en los negocios la labor de dirección es muy aparte de la producción, por lo tanto, cualquier dueño debe tener las dos labores diferenciadas o tener a personal que se encargue por separado. Al igual que la división de trabajo mejora la productividad en una planta, también debe haber una división entre las labores gerenciales y de producción. Asimismo, menciona que las personas que tienen padres que son o fueron hombres de negocio tienen la facilidad para administrar eficientemente una empresa debido a que desde pequeños han estado en contacto con ese mundo. Sin embargo, esto no se cumple cuando pasan varios años o se tienen condiciones iniciales distintas, pues el conocimiento o habilidad no son las mismas y se van perdiendo. Marshall (1890) menciona que la gestión empresarial empezó a tener importancia cuando las empresas se fueron haciendo cada vez más complejas.

Asimismo, desde comienzos del siglo XX se empezó a evaluar la administración de empresas, las personas notaron que la forma en cómo un empresario gestiona los recursos que tiene la empresa influye significativamente en los resultados obtenidos. Surgieron varios autores con ideas diversas acerca de qué aspecto enfatizar en una 
organización: Frederick Taylor, Frank y Lilian Gilbreth, Henry Fayol, Elton Mayo, entre otros. Algunos de los puntos que resaltaban era la división del trabajo, el control de los tiempos, la burocracia, los recursos humanos, etc. (Chiavenato, 1995). La gerencia de una empresa se fue convirtiendo en un tema cada vez más estudiado.

\subsection{Evidencia empírica}

Hay varios autores que han escrito acerca de la capacitación a empresarios. A pesar de no referirse a microempresarios en general se pueden obtener algunas luces acerca de lo encontrado.

O'Connell \& Byrne (2012) realizaron una investigación acerca de los determinantes y efectos de la capacitación en los trabajadores de una empresa usando la NCPP/ESRI Changing Worplace Survey, una encuesta que recoge información acerca del mercado laboral de Irlanda. Plantean un modelo logit en el cual descubren que los determinantes de la capacitación son la edad, educación, tipo de contrato, tenencia del local y tamaño de la firma. En el estudio se ve que las personas con grado de educación secundaria o universitaria estaban mejor posicionadas para recibir capacitación que las personas con menor nivel educativo.

Por otro lado, Frazis, Gittleman y Joyce (1998) toman un enfoque diferente al analizar la intensidad de la capacitación y no solo la incidencia. Se usa la encuesta Survey of Employer-Provided Training (de Estados Unidos) y luego de utilizar un modelo probit se tiene como resultado que las variables: tamaño de la empresa, presencia de un sindicato, número de beneficios y tipo de contrato, afectan a la decisión de capacitar a los trabajadores. Además, un resultado muy robusto fue que los lugares donde se ofrecen beneficios o que innovan en sus prácticas tienen más probabilidad de dar capacitación a los empleados. Adicionalmente, mencionan como hecho estilizado que las empresas grandes suelen proveer más capacitación que las pequeñas, utilizando en su estudio una muestra de empresas de más de 50 empleados.

Barron, Black y Lowenstein (1987) ofrecen una explicación para la influencia del tamaño de la empresa sobre la capacitación. Ellos argumentan que los negocios de mayor tamaño ofrecen más capacitación a sus trabajadores debido a que éstos enfrentan costos de monitoreo mayores que las pequeñas empresas y que una manera de economizar es a través del entrenamiento y contratando mejores trabajadores (aunque mencionan que se 
puede dar el caso en que se prefiera contratar trabajadores más calificados y de esa forma no tener que capacitar). El análisis, que se realizó mediante un modelo logit dio como resultado que empleadores de firmas grandes tienden a contratar en mayores cantidades personal ya capacitado y ofrecen más capacitación.

Oosterbeck (1998) analizó los factores que influyen en el resultado de que un trabajador se capacite. En el estudio se distinguieron las características asociadas a la empresa y al trabajador que pueden afectar al resultado final. Se aplicó un modelo probit para la encuesta International Adult Literacy Survey, la cual obtiene información de 22 países de diferentes continentes, principalmente europeos, y se obtuvo como resultado que las variables más importantes son el género, edad, características propias del trabajador y el área de actividad económica al que pertenece la empresa. Un resultado interesante del autor es que encuentra que las personas con mayores responsabilidades en casa o que son cabezas del hogar tienen mayores probabilidades de ser capacitadas. Una posición diferente es la de Ypeij (2006) que en un libro acerca de las diferencias en la acumulación de capital de acuerdo al género, menciona que las mujeres, al tener mayor responsabilidad sobre el cuidado de los hijos y en el hogar, tienen mayores costos asociados a dedicar más tiempo en el trabajo.

Por otro lado, Palacios (2013) realiza un trabajo acerca de los determinantes de la capacitación para Chile usando la Encuesta Laboral. En el estudio se utilizan un modelo de regresión lineal y otro de variable dependiente limitada debido a que se estudia tanto la proporción de trabajadores que han sido capacitados como el haber sido capacitado o no. Se encuentra que las variables que más influyen son el rubro de la empresa, tamaño de la empresa, la existencia de un sindicato, la remuneración e incentivos laborales. Respecto al rubro de la empresa, el autor encuentra que ciertos sectores tienen la mayor proporción de personal capacitado. Los "incentivos laborales" es un variable proxy del deseo de las empresas de mantener relaciones de largo plazo con los trabajadores, por lo que es lógico que en el resultado de la investigación la variable tenga un signo positivo.

Respecto a la efectividad de la capacitación, Karlan \& Valdivia (2011) así como Monge-González, Rodríguez-Álvarez y Torrentes-García (2013) realizaron estudios en los cuales se proveía de capacitación a un grupo de microempresarios, dueños de negocios, clientes de una microfinanciera peruana y otra costarricense, respectivamente. Luego de la intervención se procedió a recoger los datos de las empresas de la muestra y mediante técnicas como "diferencias en diferencias" y un "modelo de variable 
dependiente limitada" se comprobó en ambos casos que la capacitación tuvo resultados positivos en las prácticas empresariales y en las ventas.

Por otro lado, O'Connell \& Byrne (2012) analiza el efecto de la capacitación sobre el salario de los trabajadores empleando un modelo de regresión lineal y un propensity score matching (PSM) con datos de empresas de Irlanda. Se usó el primer modelo para ver cómo cambiaba el salario al ser capacitado, luego, para controlar por el sesgo producido por la heterogeneidad del grupo de personas que se capacitaron y las que no lo hicieron, se utilizó el PMS dando resultados similares a los obtenidos en el primer modelo. Se encontró que los salarios de las personas capacitadas eran mayores que el resto.

Bloom et al. (2013) realizaron una investigación acerca de los temas de capacitación en la India. Se capacitó a empresarios en la industria textil en prácticas administrativas básicas acerca de la calidad, inventarios y eficiencia operacional. Los resultados obtenidos fueron de una mejora en la productividad asociada a las mejores prácticas en gestión. Los autores afirman que la razón para que tales capacitaciones sean tan efectivas y tan poco difundidas son las barreras en la difusión de información acerca de los programas de capacitación.

A diferencia de los autores previamente citados, en el presente trabajo se estudiará las características de los empresarios de MYPE que desean capacitarse. Debido que es común la existencia de empresas donde el dueño es el mismo trabajador, el estudio incluirá variables relacionadas a los trabajadores y a la empresa. Para cumplir con este objetivo se usará un modelo logit a semejanza de varios autores en la literatura. Asimismo, se evaluará la efectividad de la capacitación para el aumento de los ingresos de las empresas. Debido a la posible heterogeneidad entre el grupo de personas capacitadas con el grupo de personas no capacitadas se plantea el uso de un PSM al igual que O'Connell \& Byrne (2012). 


\section{CAPÍTULO III: EVALUACIÓN EMPÍRICA}

\subsection{Descripción de fuente de datos}

Para el presente trabajo se usará la base de datos EMYPE. Esta encuesta tiene representatividad a nivel de ciudades grandes las cuales son elegidas por el INEI debido a su relevancia económica, estas son: Arequipa, Chiclayo, Huancayo, Lima, Maynas, Piura, Trujillo, Cusco, Huamanga y San Román.

Las empresas fueron clasificadas de acuerdo a la ley vigente y además tienen la característica de ser todas manufactureras (algunas de actividad más compleja que otras). La encuesta se realizó preguntándole al personal de la empresa acerca de asociatividad con otras empresas, capacitación empresarial, equipos, acceso al sistema financiero, ingresos percibidos por la actividad, etc. Se realizó la encuesta del año 2010 al 2013, si bien conforme se volvió a ejecutar la encuesta algunas preguntas fueron añadidas y otras eliminadas no interfiere para el presente estudio pues la mayoría de variables de interés se encuentran en todas las encuestas. A pesar de tener disponible la EMYPE de cuatro años solo se usarán del año 2011 al 2013 debido a que la información del 2010 se encuentra incompleta. Las observaciones son cuantiosas y permitirán tener los suficientes grados de libertad para hacer generalizaciones.

\section{Tabla 3.1}

Número de observaciones por encuesta EMYPE

\begin{tabular}{|c|c|}
\hline Año & Observaciones \\
\hline 2013 & 3,023 \\
\hline 2012 & 5,410 \\
\hline 2011 & 5,435 \\
\hline
\end{tabular}

Fuente: INEI $(2013,2012,2011)$.

\subsection{Descripción de las variables}

Las variables seleccionadas se eligieron de acuerdo a la teoría y disponibilidad de ellas en la EMYPE, asimismo, se procedió a crear nuevas variables a partir de las que existían en la encuesta pues de otra forma el análisis no sería posible. A continuación, se presenta un análisis descriptivo de cada variable que se utilizará junto con el procedimiento para la creación de las nuevas variables. 


\subsubsection{Variables continuas}

Lo más resaltante de estos estadísticos son los valores que toman las variables referidas a la utilidad operativa y a los trabajadores de la empresa, pues tienen valores muy extremos respecto de la media. Para lidiar con estos valores atípicos se usarán las metodologías estadísticas: residuos de Pearson, desviaciones residuales y Dbeta de Pregibon.

Tabla 3.2

Estadísticos de las variables edad, utilidad operativa, antigüedad y trabajadores de la empresa

\begin{tabular}{|l|cccc|}
\hline \multicolumn{1}{|c|}{ Estadísticos } & Edad & $\begin{array}{c}\text { Utilidad } \\
\text { Operativa }\end{array}$ & $\begin{array}{c}\text { Antigüedad de la } \\
\text { empresa }\end{array}$ & $\begin{array}{c}\text { Trabajadores de } \\
\text { la empresa }\end{array}$ \\
\hline Media & 44.65 & 102,304 & 9.83 & 7.58 \\
Máximo & 90 & $12,200,000$ & 69 & 990 \\
Mínimo & 18 & $-1,066,005$ & 0 & 0 \\
Desviación Estándar & 11.59 & 302,734 & 7.69 & 17.14 \\
Coef. de Variación & 0.26 & 2.96 & 0.78 & 2.26 \\
\hline
\end{tabular}

Fuente: INEI $(2013,2012,2011)$.

La edad media de los informantes de la encuesta es de 44.65 años por lo que se espera que el signo que acompañe al coeficiente sea negativo pues la rentabilidad de la capacitación va disminuyendo conforme se acerca la jubilación. Asimismo, se espera que el signo de la antigüedad de la empresa sea positivo pues mientras más tiempo lleve significaría que se planea mantener en el largo plazo, por lo tanto se requeriría capacitación. El signo de la variable de los trabajadores de la empresa posiblemente sea positivo, pues es una proxy del tamaño de la empresa.

\subsubsection{Variables categóricas}

La pregunta que se hace para saber si una persona ha recibido capacitación es "Durante el año___ ¿ ¿asistió usted o el responsable de la empresa a algún evento de capacitación relacionado a la Gestión Empresarial?". La relación entre las personas que contestaron sí y no es asimétrica lo que podría justificar el uso de una función log-log complementaria y no una logística para la distribución de las probabilidades que se está asumiendo, por lo tanto, teniendo en cuenta ello se presentarán los resultados con ambas funciones. 
Tabla 3.3

Frecuencias de las variables capacitación empresarial, género, licencia municipal, cantidad de equipos y actividad empresarial

\begin{tabular}{|l|cc|}
\hline \multicolumn{1}{|c|}{ Variables / Respuesta } & Frecuencia & Porcentaje \\
\hline Capacitación hace un año & & \\
Sí & 2,113 & 18.7 \\
No & 9,186 & 81.3 \\
Total & $\mathbf{1 1 , 2 9 9}$ & $\mathbf{1 0 0}$ \\
\hline Género & & \\
Masculino & 7,204 & 63.8 \\
Femenino & 4,095 & 36.2 \\
Total & $\mathbf{1 1 , 2 9 9}$ & $\mathbf{1 0 0}$ \\
\hline Licencia Municipal & & \\
Sí & 5,815 & 81.3 \\
No & 1,338 & 18.7 \\
Total & $\mathbf{7 , 1 5 3}$ & $\mathbf{1 0 0}$ \\
\hline Cantidad de equipos & & \\
Bajo la mediana & 5,255 & 46.5 \\
Sobre la mediana & 6,044 & 53.5 \\
Total & $\mathbf{1 1 , 2 9 9}$ & $\mathbf{1 0 0}$ \\
\hline Actividad empresarial & & \\
Complejidad básica & 9,281 & 66.9 \\
Complejidad no básica & 4,587 & 33.1 \\
Total & $\mathbf{1 3 , 8 6 8}$ & $\mathbf{1 0 0}$ \\
\hline
\end{tabular}

Fuente: INEI $(2013,2012,2011)$.

El signo de la variable género se espera que sea positivo cuando se usa como una variable indicador para el hombre debido a los resultados de Ypeij (2006), sin embargo, cabe resaltar que los estudios propiamente de capacitación empresarial mencionados anteriormente no encontraron relación alguna. Por otro lado, la variable de licencia municipal es una proxy de la formalidad de la empresa, se espera que su signo sea positivo debido a que es una señal de querer mantener la empresa en el largo plazo y además de sentirse empoderado del negocio.

Las variables referidas al capital de la empresa y la actividad empresarial se construyeron a partir de otras existentes. En la EMYPE se pregunta acerca de la tenencia de ocho tipos de equipos: Computadora de escritorio, computadora portátil, impresora, escáner, proyector, fotocopiadora, multifuncional y otros ${ }^{1}$. Se procedió a sumar todos los

\footnotetext{
${ }^{1}$ En la EMYPE del 2013 se incluyó una pregunta acerca de la tenencia de "Teléfono con acceso a internet", para guardar consistencia con encuestas pasadas se consideró este equipo como parte de "Otros".
} 
equipos y clasificar aquellos que tengan un número de equipos sobre y debajo de la mediana de forma de tener una variable indicador acerca del capital de la empresa. Con esta metodología se está asumiendo que los equipos tienen un peso igual. Se espera que el signo de esta variable sea positivo pues mientras más equipos se tenga se necesita personal capacitado para manejarlos.

Asimismo, si bien todas las empresas pertenecen al sector de manufactura, se distinguió entre la complejidad de sus procesos mediante una variable indicadora. Esta variable toma el valor de 1 si es que la complejidad de los procesos se considera básica y 0 en los demás casos. La clasificación se hizo de acuerdo a los subsectores de manufactura en donde las empresas operan. Se espera que la variable tenga un signo negativo.

Tabla 3.4

Nivel de educación del informante

\begin{tabular}{|l|cc|}
\hline \multicolumn{1}{|c|}{ Educación } & Frecuencia & Porcentaje \\
\hline Sin nivel & 22 & 0.19 \\
Inicial & 1 & 0.01 \\
Primaria incompleta & 97 & 0.86 \\
Primaria completa & 304 & 2.69 \\
Secundaria incompleta & 342 & 3.03 \\
Secundaria completa & 2,474 & 21.89 \\
Superior no univ. incompleta & 439 & 3.88 \\
Superior no univ. completa & 1,497 & 13.25 \\
Superior univ. incompleta & 1,108 & 9.81 \\
Superior univ. completa & 5,016 & 44.39 \\
\hline \multicolumn{1}{|c|}{ Total } & $\mathbf{1 1 , 3 0 0}$ & $\mathbf{1 0 0}$ \\
\hline
\end{tabular}

Fuente: INEI $(2013,2012,2011)$.

El signo de la variable educación se espera positivo, pues las ganancias de capacitación conforme más educación se tenga son mayores. La información es presentada en categorías. En este caso se está asumiendo que vale más una educación superior universitaria a una no universitaria.

Se incluirán variables de tipo indicadoras de cada ciudad en particular. El signo esperado de las variables indicadores cuando se comparen contra Lima no es claro, pues puede ser positivo si prima el hecho de que al estar en lugares lejanos a la capital se carezca de beneficios o educación en la materia, por lo que su demanda sería mayor, por otro lado, al estar en un mercado menos desarrollado, la necesidad de capacitarse para sobrevivir en el largo plazo es menor, en este caso el signo sería negativo. 
Tabla 3.5

Provincia de la empresa encuestada

\begin{tabular}{|c|c|c|}
\hline Provincia & Frecuencia & Porcentaje \\
\hline Arequipa & 2,515 & 18.14 \\
\hline Chiclayo & 1,142 & 8.23 \\
\hline Huancayo & 926 & 6.68 \\
\hline Lima & 5,452 & 39.31 \\
\hline Maynas & 826 & 5.96 \\
\hline Piura & 716 & 5.16 \\
\hline Trujillo & 2,041 & 14.72 \\
\hline Cusco & 102 & 0.74 \\
\hline Huamanga & 48 & 0.35 \\
\hline San Román & 100 & 0.72 \\
\hline+2 & 13,868 & 100 \\
\hline
\end{tabular}

Fuente: INEI (2013, 2012, 2011).

\subsection{Evaluación empírica}

El primer modelo a utilizar es el de variable dependiente limitada. Estos tipos de modelo requieren hacer un supuesto acerca de la distribución de la variable a estimar. En el presente estudio se asume una distribución logística, por ser una de las más usadas en estudios similares. En adelante, el modelo de variable dependiente limitada se llamará "logit" por la función de distribución asumida. Estos modelos se estiman mediante una función de máxima verosimilitud.

El segundo modelo es de evaluación de impacto. El gran reto de la evaluación de impacto es encontrar una observación comparable a la que recibió el tratamiento que se pretende estudiar. Para esto hay diferentes modelos como los basados en regresión que intentan resolver el problema mediante la media condicionada del resultado del tratamiento, otros que ponderan a las observaciones que tienen una probabilidad estimada más pequeña o alta en comparación con el resto, otros que son los llamados de vecinos más próximos que a través de similitudes de las observaciones usando variables control. Se eligió el PSM, uno de los métodos de vecinos más próximos, debido a que este requiere estimar la probabilidad de recibir el tratamiento, lo cual ya estaría cubierto con el primer modelo, por lo que la aplicación del segundo modelo es automática.

\subsubsection{Modelo logit}

Se estimó un modelo logit con la base de datos mencionada, se realizó un pool de datos para tener más grados de libertad. Es importante resaltar que la variable de licencia 
municipal solo se encuentra en las encuestas del 2012 y 2013, sin embargo, los resultados al excluirla teniendo un pool de los tres años no cambia en gran manera los parámetros estimados. Asimismo, las provincias de Cusco, San Román y Huamanga solo aparecen en el 2013, por ello no se les incluyó en la estimación. Por último, el modelo se realizó solo para las observaciones en donde el informante es el conductor de la empresa, gerente o administrador, pues ellos tienen de decisión sobre la empresa y guiarla de acuerdo a lo que ha aprendido a diferencia de un trabajador común.

Figura 3.1

Ecuación logit

$$
E\left[\text { Capacitación } n_{i}=1 \mid X=x_{i}\right]=\frac{\exp \left\{X^{\prime} \beta\right\}}{1+\exp \left\{X^{\prime} \beta\right\}}
$$

Donde $X_{i}$ es un vector de variables que contiene las variables referidas al género, edad y educación del informante; una variable para diferenciar la actividad empresarial, tenencia de licencia municipal, antigüedad de la empresa, una variable indicadora de tenencia de equipos sobre la mediana de la muestra, provincia de origen, cantidad de trabajadores de la empresa y año de la muestra. El vector $\beta$ recoge el cambio marginal del logaritmo natural de la razón de momios ante un cambio en una unidad de alguna variable contenida en el vector $X_{i}$. Esta última definición no es muy intuitiva y para motivos del estudio no es relevante interpretarla, sino la significancia de cada uno de esos parámetros para explicar la probabilidad de haber sido capacitado. Por último, en vez de asumir una distribución logística se probó una función log-log complementaria, debido a que esta permite lidiar con probabilidades asimétricas.

Figura 3.2

Ecuación log-log complementaria

$$
E\left[\text { Capacitación } n_{i}=1 \mid X=x_{i}\right]=1-\left(\frac{1}{\exp \{1\}}\right)^{\exp \{\mathrm{X} \prime \beta\}}
$$


Tabla 3.6

Resultados del primer modelo econométrico

\begin{tabular}{|c|c|c|c|}
\hline Variables & $\begin{array}{l}\text { M. función de dist. } \\
\text { logística }\end{array}$ & $\begin{array}{l}\text { M. función de dist. log- } \\
\text { log complementaria }\end{array}$ & $\begin{array}{l}\text { M. sin la variable } \\
\text { licencia municipal }\end{array}$ \\
\hline \multirow{2}{*}{ Constante } & $-3.29 * * *$ & $-3.239 * * *$ & $-3.396^{* * *}$ \\
\hline & -11.91 & -13.08 & -15.47 \\
\hline \multirow{2}{*}{ Género } & -0.006 & -0.001 & -0.013 \\
\hline & -0.08 & -0.02 & -0.21 \\
\hline \multirow{2}{*}{ Edad } & $-0.013 * * *$ & $-0.012 * * *$ & $-0.012 * * *$ \\
\hline & -3.98 & -4.00 & -4.78 \\
\hline \multirow{2}{*}{ Educación } & $0.148 * * *$ & $0.135 * * *$ & $0.176^{* * *}$ \\
\hline & 6.92 & 6.96 & 10.21 \\
\hline \multirow{2}{*}{ Actividad Empresarial } & -0.021 & -0.012 & -0.052 \\
\hline & -0.28 & -0.19 & -0.85 \\
\hline \multirow{2}{*}{ Licencia Municipal } & $0.390 * * *$ & $0.346^{* * *}$ & \\
\hline & 3.69 & 3.60 & \\
\hline \multirow{2}{*}{ Antigüedad de la Empresa } & -0.004 & -0.004 & -0.002 \\
\hline & -0.91 & -0.86 & -0.58 \\
\hline \multirow{2}{*}{ Cantidad de equipos } & $0.877 * * *$ & $0.798 * * *$ & $0.904 * * *$ \\
\hline & 10.72 & 10.84 & 13.81 \\
\hline \multirow{2}{*}{ Arequipa } & $0.218 * *$ & $0.178 * *$ & $0.164 * *$ \\
\hline & 2.24 & 2.10 & 2.04 \\
\hline \multirow{2}{*}{ Chiclayo } & $0.569 * * *$ & $0.485 * * *$ & $0.559 * * *$ \\
\hline & 4.12 & 4.06 & 5.10 \\
\hline \multirow{2}{*}{ Huancayo } & $0.628 * * *$ & $0.544 * * *$ & $0.736^{* * *}$ \\
\hline & 4.83 & 4.93 & 7.14 \\
\hline \multirow{2}{*}{ Maynas } & $0.417 * *$ & $0.353 * *$ & $0.511 * * *$ \\
\hline & 2.52 & 2.46 & 4.04 \\
\hline \multirow{2}{*}{ Piura } & $0.535^{* * *}$ & $0.469 * * *$ & $0.420 * * *$ \\
\hline & 3.37 & 3.46 & 3.30 \\
\hline \multirow{2}{*}{ Trujillo } & 0.171 & 0.139 & $0.380 * * *$ \\
\hline & 1.50 & 1.38 & 4.41 \\
\hline \multirow{2}{*}{$\begin{array}{l}\text { Trabajadores de la } \\
\text { Empresa }\end{array}$} & $0.014 * * *$ & $0.009 * * *$ & $0.017 * * *$ \\
\hline & 3.77 & 3.78 & 5.90 \\
\hline \multirow{2}{*}{ Año 2013} & $0.274 * * *$ & $0.242 * * *$ & $0.340 * * *$ \\
\hline & 3.52 & 3.59 & 4.38 \\
\hline \multirow{2}{*}{ Año 2012} & & & 0.097 \\
\hline & & & 1.54 \\
\hline $\mathrm{N}^{\circ}$ Observaciones & 5,704 & 5,704 & 9,310 \\
\hline Likelihood-ratio & 392.87 & 391.04 & 673.04 \\
\hline
\end{tabular}

*Nivel de significancia: $* * * 0.01, * * 0.05, * 0.10$

*Los número en cursiva corresponden al estadístico Z. 
Para probar la bondad de ajuste de los modelos de variable dependiente hay diversos métodos y test. Al usar el Likelihood-Ratio se ve que el modelo tiene buen ajuste. Sin embargo, el pseudo $\mathrm{R}^{2}$ de McFadden resulta muy pequeño en los dos primeros modelos (menos de 0.08), a pesar de ser utilizado este último estadístico, se desestima su uso pues en un capítulo del libro de Hosmer \& Lemeshow (2000) se demuestra que un modelo puede tener un bajo pseudo $\mathrm{R}^{2} \mathrm{y}$ sin embargo puede dar buenos resultados. Estos mismos autores tienen un test para evaluar estos modelos que se basa en dividir la muestra por grupos y calcular el valor esperado de recibir el tratamiento, luego con las probabilidades estimadas calcular el promedio según los grupos definidos y ver si están cerca. A pesar de ser un test con bastante intuición tiene dos problemas: la elección del número de grupos y la sensibilidad del test al incluir diferentes variables. Se hizo una simulación para cada uno de los grupos posibles y efectivamente el resultado cambio de acuerdo al número de grupos ${ }^{2}$.

Una metodología para determinar el ajuste es el porcentaje correctamente clasificado. Este determina un umbral para redondear hacia 1 las probabilidades estimadas mientras que las que caen por debajo se redondean a 0 , luego se calcula el porcentaje en el cual las probabilidades redondeadas a 1 o 0 acertaron con las variables observadas. Se realizó el procedimiento para cada uno de los umbrales posibles y se obtuvieron los siguientes resultados.

En la figura de porcentaje correctamente clasificado se ve que con un umbral de 0 se clasifica correctamente a un $20 \%$ aproximadamente. Este porcentaje crece muy fuertemente al subir tan solo un poco el umbral y se mantiene en un poco más en $80 \%$ pronto, lo que nos habla de un buen ajuste del modelo.

\footnotetext{
${ }^{2} \mathrm{Si}$ bien la gran mayoría de los test dieron como resultado un buen ajuste, no se vuelve un criterio para discriminar un modelo.
} 
Figura 3.3

Porcentaje de observaciones correctamente calificadas según el modelo

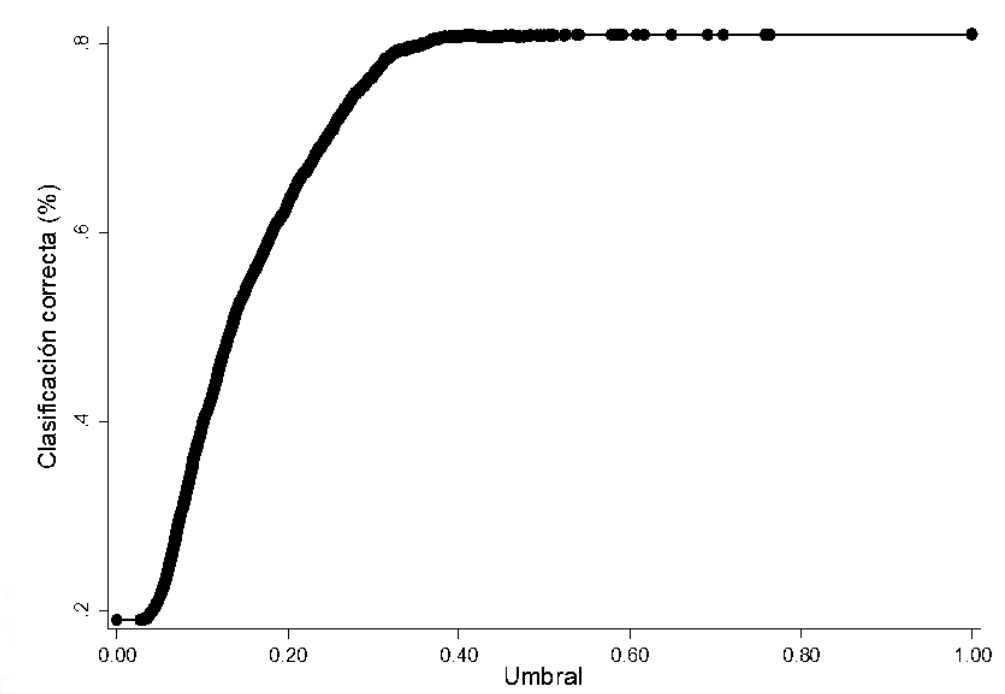

Asimismo, otro test parecido al anterior es el de la curva Receiver Operating Characteristics (curva ROC). En este se grafica el nivel de sensitividad (porcentaje de veces que se clasifica correctamente las respuestas afirmativas) contra el complemento del nivel de especificidad (porcentaje de veces que se clasifica correctamente las respuestas negativas). Se compara esta curva contra una recta de 45 grados para ver si respecto a las veces que se equivoca en clasificar a las respuestas negativas acierta en mayor proporción las respuestas afirmativas.

Figura 3.4

\section{Curva ROC}

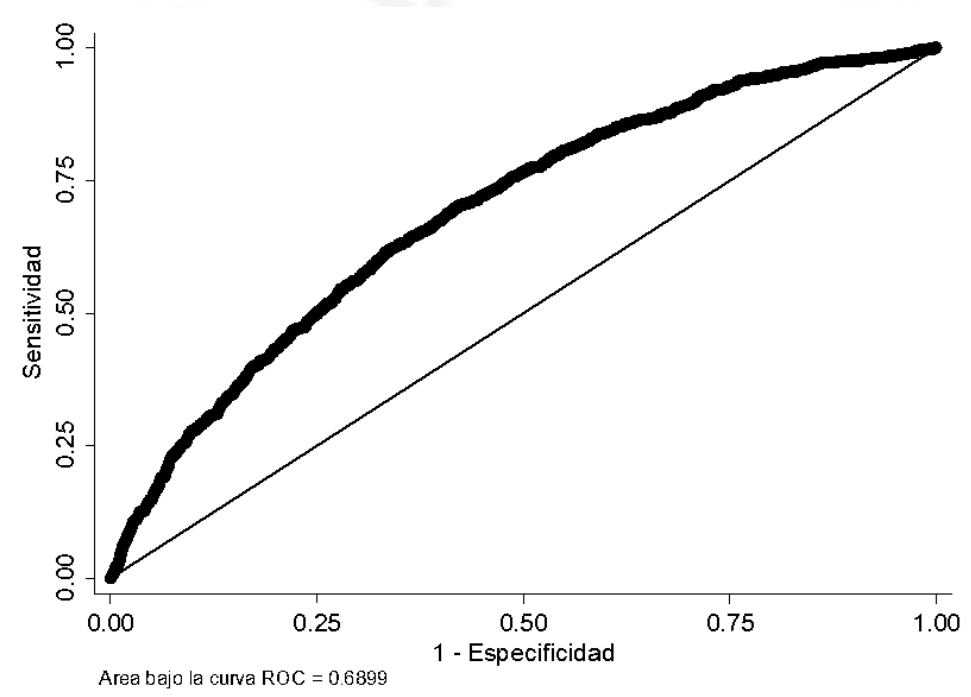


Se puede ver que el área bajo la curva ROC es casi el 70\% de lo posible, lo que se considera un porcentaje aceptable para un modelo econométrico.

Como se ve en la tabla de resultados no hay una gran diferencia cuando se usa una función log-log complementaria a una logística, asimismo, la pérdida de observaciones al usar la variable licencia municipal no altera de gran manera los resultados. Es interesante que en el tercer modelo se obtuviera una estimación aproximadamente igual a 1, lo que da más soporte a la elección del PSM para el segundo modelo, pues tener valores de 1 o muy cercanos a 0 o 1 representan un problema al usar los métodos basados en ponderaciones de acuerdo a sus probabilidades.

Luego de realizar estas pruebas de bondad de ajuste se procedió a comprobar la existencia de datos atípicos en la muestra que pudieran distorsionar los resultados. Se han usado dos metodologías, la primera es analizar los residuos de la estimación y la segunda analizar cuán importante es una observación para la estimación de los parámetros del modelo.

Se calcularon los residuos de Pearson y las desviaciones residuales del modelo y se graficaron contra las observaciones.

Figura 3.5

Residuos de Pearson

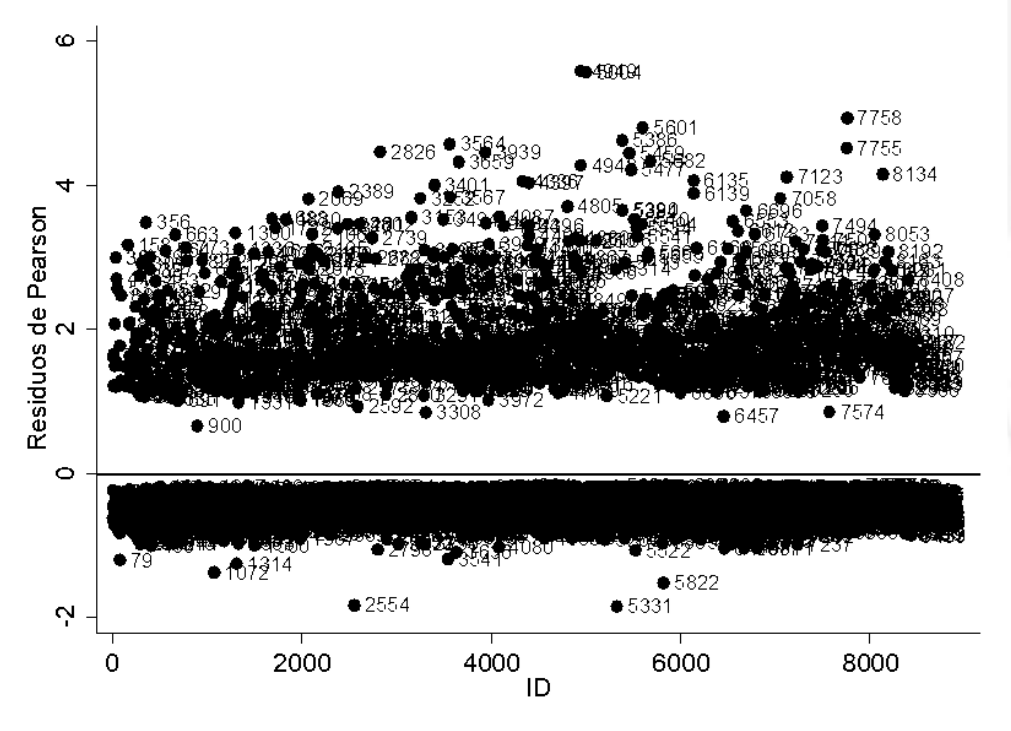


Figura 3.6

Desviaciones residuales

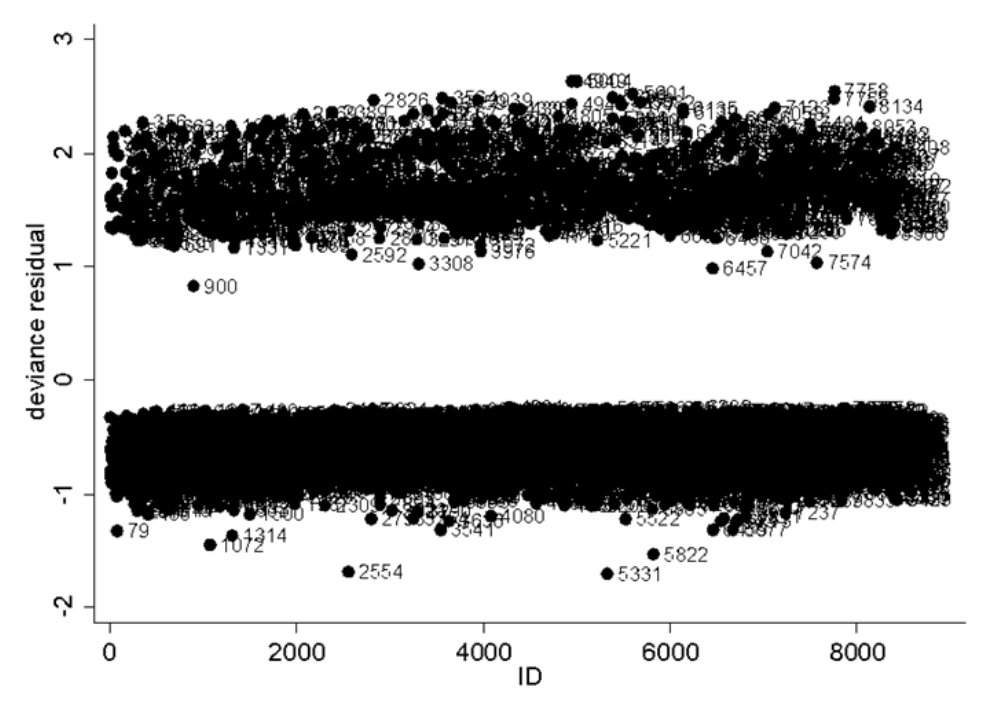

Como se puede ver, las observaciones que causan problemas son 2554, 4949, 5004 y 5331. Sin embargo, al removerlas y volver a estimar el modelo no existen grandes cambios, por lo que se determina incluirlas en el modelo.

En el siguiente paso se calculó el dbeta de Pregibon y se graficó contra las observaciones.

Figura 3.7

Dbeta de Pregibon

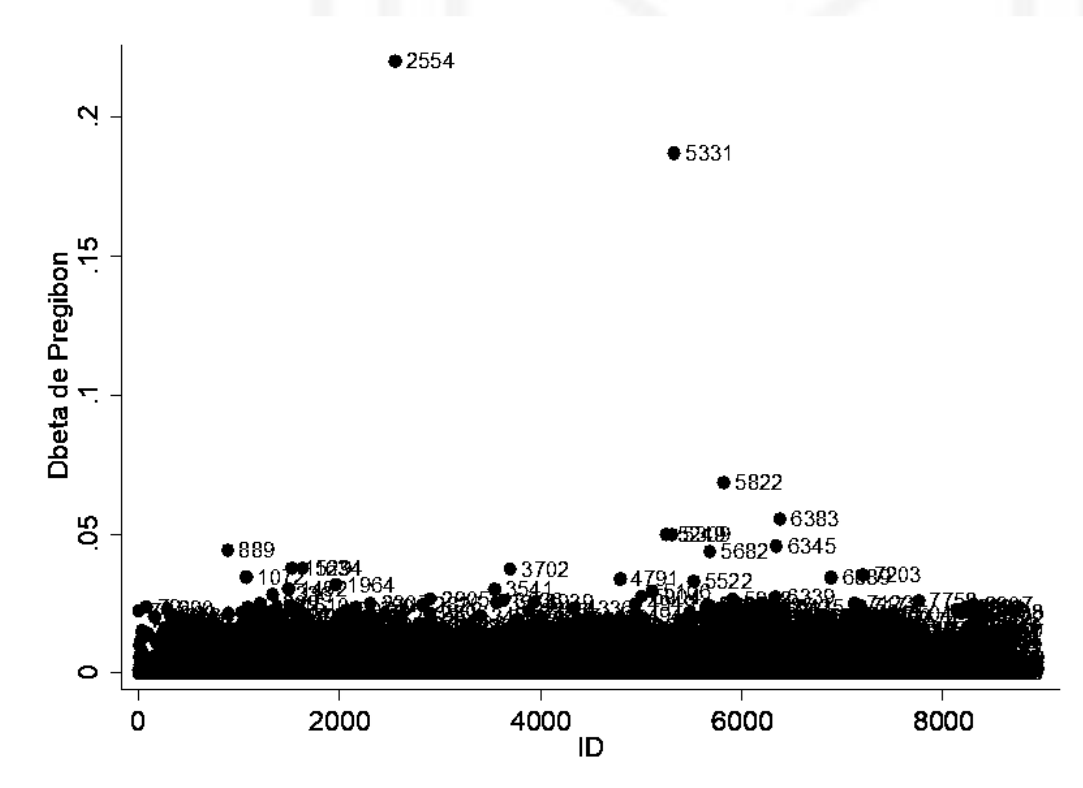


El gráfico nos muestra que las observaciones que influencian son 2554, 5331 y 5822. Sin embargo, al igual que en el anterior caso la eliminación de estas observaciones no influye en la estimación de los parámetros. Por lo tanto, se decide mantenerlas en la estimación final.

Al ver los resultados de la regresión no se puede afirmar fehacientemente si es que priman las variables referidas a la empresa o al empresario, pues si bien el mayor estadístico $\mathrm{Z}$ lo tiene la variable referida a cantidad de equipos, la segunda variable más importante es la educación y de esta forma la importancia se va intercalando entre las variables referidas a las características de la empresa o empresario.

Por otro lado, es interesante constatar que en ningún caso la variable "Género" resulta significativa. Se esperaba que el signo sea positivo si la variable binaria estaba codificada de modo que 1 sea igual a hombre, sin embargo, y de acuerdo a trabajos anteriores, el género no parece ser importante para capacitarse o no.

Otro resultado a resaltar es que la importancia de la variable dummie de la provincia de procedencia es menor en los lugares más desarrollados. El resultado no va acorde con lo señalado por Bloom et. al (2010) pues estos lugares deberían tener mayor competencia y por lo tanto la demanda de capacitación debería ser mayor para equiparar la ventaja competitiva de las empresas que son bien manejadas. Sin embargo, el resultado puede ser explicado por los retos de estas provincias, pues al estar en un entorno menos favorable para el crecimiento de la empresa, los dueños o gerentes buscarían formas de mejorar el manejo y tener más posibilidades de sobrevivir y generar un retorno mayor.

\subsubsection{Propensity score matching}

Una vez obtenida la estimación final del modelo logit se puede proceder a realizar la evaluación de impacto mediante el método de propensity score matching. El método consiste en comparar las utilidades operativas de un empresario que se haya capacitado con las obtenidas por un empresario que no lo hizo. Por ello, se requieren las probabilidades de capacitación, para emparejar a empresarios que sean parecidos, pero que se diferencien en su capacitación. En otras palabras, comparamos la utilidad operativa de alguien que se capacitó versus las utilidades operativas de alguien que tuvo las mismas oportunidades de capacitarse que el primero, pero no lo hizo. Este proceso se continúa para el resto de individuos de la muestra. La comparación final de las diferencias 
obtenidas de las sucesivas comparaciones se evalúa mediante un test de medias. Antes de estimar el efecto del tratamiento se procedió a eliminar valores atípicos en la variable de utilidad operativa.

Figura 3.8

Utilidad operativa antes del tratamiento de datos atípicos

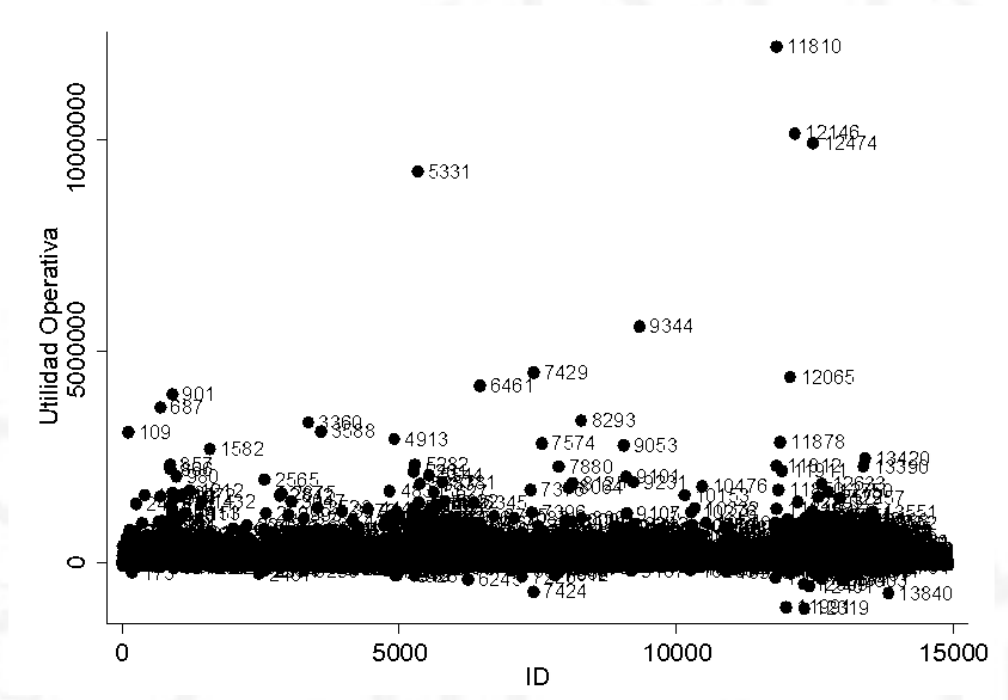

En el gráfico se ve que hay diversas observaciones que alterarían la estimación. Se eligió quedarse con los datos entre el percentil 1 y el 99, de esta forma se tiene un método simple pero menos subjetivo que retirar las variables basándose en un gráfico. El resultado fue el siguiente.

Figura 3.9

Utilidad operativa luego del tratamiento de datos atípicos

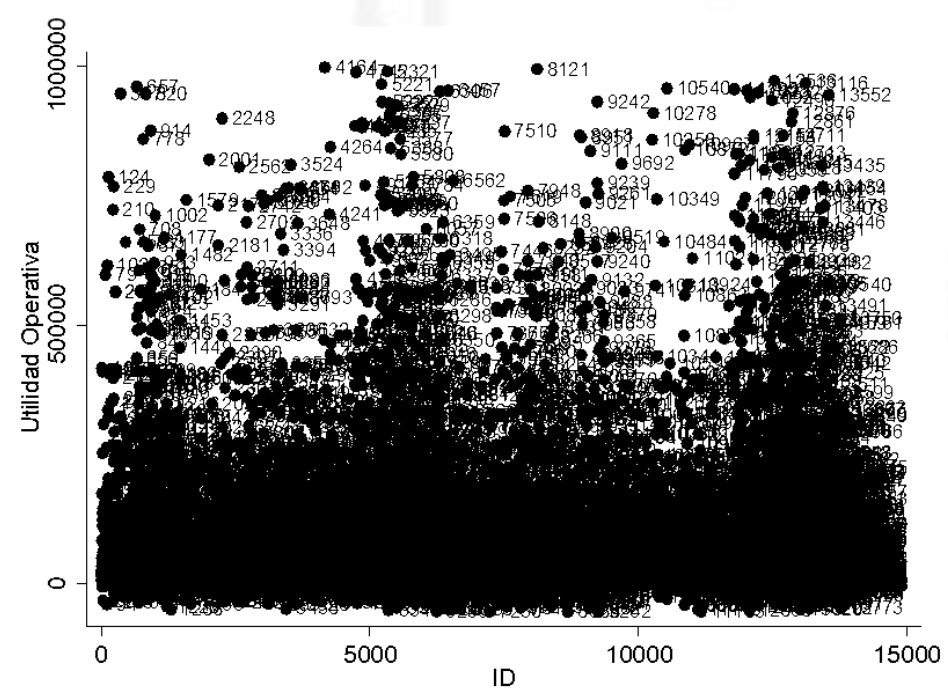


Se consideró que esta nueva muestra no generaría mayor problema en el análisis de los datos, por lo que se procedió a estimar el efecto del tratamiento.

Tabla 3.7

Estimación del efecto de la capacitación sobre las utilidades operativas

\begin{tabular}{cccc}
\hline Coeficiente & Error Estándar & Estadístico Z & Intervalo de Confianza al 95\% \\
\hline 176.47 & 6448.41 & 0.03 & {$[-12462.18-12815.11]$} \\
\hline
\end{tabular}

Como se puede apreciar en la tabla, los resultados muestran que no se puede rechazar la hipótesis que el tratamiento no tenga efectos significativos sobre la utilidad operativa de la empresa, debido a que el estadístico $\mathrm{Z}$ se encuentra muy lejos de los valores críticos de la distribución (aproximadamente 1.96).

Es importante mencionar que los resultados muestran un coeficiente positivo, el cual se interpreta como el monto adicional de utilidades operativas que tendría un empresario capacitado. Sin embargo, el error estándar es bastante alto, lo cual hace que el coeficiente no pase la prueba de significancia. La alta dispersión se debe a que en la encuesta utilizada no se distingue entre la calidad de la capacitación recibida, incluso si es que el empresario llegó a utilizar los conocimientos aprendidos en esta. Esto explicaría porque en la literatura se encuentran resultados positivos al realizar evaluaciones de impacto con grupos reducidos y controlados.

De acuerdo a Mature et al. (2008) existe una falta de supervisión de las personas capacitadas una vez concluido el servicio. Esto puede originar la persistencia de capacitaciones que no tienen impacto en el negocio de los empresarios. En la muestra utilizada para evaluar la efectividad de la capacitación no se tiene información acerca de la ejecución de lo aprendido. Este factor incrementa la heterogeneidad del coeficiente, haciéndolo no significativo. 


\section{CAPÍTULO IV: ORGANIZACIÓN DE LAS CAPACITACIONES}

Para analizar la oferta de capacitación, se realizó una entrevista a un funcionario de la subgerencia de Fomento de la Inversión y Turismo de una Municipalidad de Lima Metropolitana. En esta se habló acerca de las funciones de la municipalidad y la forma en cómo se promueve la capacitación de los microempresarios. Asimismo, se asistió a capacitaciones empresariales para obtener información de primera mano acerca del desarrollo de las mismas.

En la municipalidad, la capacitación microempresarial se encuentra dentro de las metas establecidas en el Texto Único de Procedimientos Administrativos (TUPA), por ello, organizar este tipo de eventos es un objetivo constante dentro de la subgerencia. Las capacitaciones se realizan en el auditorio de la Municipalidad que cuenta con un aforo de 500 personas, asimismo, se organizan otros eventos dentro del distrito debido a su gran extensión geográfica.

Existe un nexo entre la municipalidad y las instituciones colaboradoras que son quienes brindan la capacitación, hay veces en las que la misma municipalidad se contacta con ellos y viceversa. Estas instituciones brindan capacitación en temas diversos de acuerdo a la especialidad de la organización. Entre las instituciones que realizan las capacitaciones se encuentran universidades, organizaciones no gubernamentales y empresas privadas.

Las razones por las cuales se brinda la capacitación son diversas dependiendo del tipo de institución encargada, por ejemplo, las universidades suelen hacerlo como proyecto de un curso, las organizaciones no gubernamentales pueden estar incentivadas por motivos filantrópicos y las empresas privadas utilizan el espacio que ganan para hacer publicidad del producto o servicio que ofrecen. Las instituciones se encargan de todos los detalles de la capacitación. Existen algunas entidades que ya tienen antecedentes brindando sus servicios y mantienen una relación de largo plazo con la municipalidad.

La municipalidad lleva un control acerca de los temas que son abordados en la capacitación, de esta forma, interviene dando el visto bueno al programa que la institución propone, luego, se realiza un monitoreo a las actividades que se realizan. Al final de cada 
capacitación se redacta un informe para mantener registro de lo acontecido, asimismo, se mantiene registro del número de asistencias.

No siempre se realiza una publicidad exhaustiva de la capacitación, pues depende de la organización entre la empresa que está brindando el servicio y la municipalidad. En caso que se llegue a realizar, se emplean diversos métodos: de manera directa, mediante comunicados a las asociaciones de microempresarios, volantes y redes sociales. Es importante mencionar que los empresarios que tienen su negocio cerca al local de la municipalidad afirman tener interés en ser capacitados, pero afirman que no tienen conocimiento de las capacitaciones que se realizan.

Luego de la entrevista, se asistió a diferentes capacitaciones brindadas por municipalidades y otras instituciones del estado. En estas se pudo observar las características de los empresarios que asistían, sus objetivos y el desarrollo de la misma capacitación.

Los horarios de las capacitaciones son variados, algunas instituciones ofrecen su servicio durante toda la mañana, mientras que otras se organizan en turnos por la noche. Las capacitaciones se dan en los locales de las instituciones. Los cuales se pueden encontrar en la oficina principal o en un lugar alquilado (por ejemplo, unas galerías comerciales). Es importante mencionar que algunas instituciones, además de este tipo de capacitaciones, también van directamente a los centros empresariales para maximizar la asistencia de las personas a las charlas. Los organizadores cuentan con una base de datos de capacitadores que van desde profesores de universidad hasta asesores de empresas. Los temas tratados suelen ser de carácter general, aunque en algunos casos se puede encontrar capacitaciones muy específicas, dirigidas a empresarios de un sector en específico. Es importante mencionar que la publicidad para promocionar las capacitaciones es primordialmente vía web, en la página de la organización y en sus respectivas cuentas en redes sociales. Los temas se enseñan a manera de exposición, algunos capacitadores utilizan material audiovisual.

Las personas que asistieron a las capacitaciones están en un rango de edad que va desde los 30 a 40 años, aproximadamente. Es importante mencionar que los eventos nunca llegan a llenar todo el lugar donde se están dando, la asistencia suele ser de $40 \%$ a $50 \%$. Algunos ya cuentan con un negocio establecido, otros trabajan de forma dependiente y hay de quienes están pensando en desarrollar un emprendimiento. Los 
asistentes colocan gran interés en las charlas e intentan aplicar los conocimientos a su negocio. Entre los empresarios, se pueden encontrar bastante diversidad entre los conocimientos con los que ya cuenta, por ejemplo, algunas de ellos entendían bien los conceptos de costeo, margen de ganancia, entre otros. Los participantes afirman que una de las limitantes en su crecimiento a través de nuevas tecnologías es la falta de capacitación y el alto monto de inversión inicial.

Es importante mencionar que antes de las capacitaciones se les pide a los empresarios colocar sus nombres, DNI, correo electrónico y alguna pregunta adicional (como la tenencia de empresa), sin embargo, no se cuenta con una base de datos estructurada que permita realizar análisis acerca del tipo de empresarios que suelen asistir. Por otro lado, las capacitaciones se centran en el momento de la charla, no se realiza ningún seguimiento a los asistentes. Esto se da debido a la dificultad de monitorear a los empresarios capacitados, pues pocos de ellos son personas recurrentes, y el problema de encontrar una medición que determine si la capacitación fue efectiva o no.

Por último, respecto a la capacitación microempresarial por páginas web, se pudo ver que se ofrece una gran variedad de temas, asimismo, a diferencia de las capacitaciones presenciales, existen recursos, cómo cuadernos de apuntes, con esquemas y gráficos que ayudan al aprendizaje del empresario. Es importante mencionar que este servicio es completamente gratuito y no se necesita registrase para tener acceso, lo cual impide la recolección de información de los empresarios que acceden al sitio web.

Como se puede ver, la capacitación microempresarial es consensuada, para ofrecer capacitación se deben pasar algunos filtros. Sin embargo, se pudo constatar que existe una falencia en la publicidad que se realiza a las capacitaciones, de otra forma, el número de asistentes podría ser mayor. Asimismo, no hay una base de datos que se maneje entre los entes capacitadores a fin de realizar análisis de las características de los participantes y el efecto de la capacitación.

Es importante mencionar que, de acuerdo a los resultados de la parte empírica del trabajo, existen características relevantes de los empresarios que se capacitan, por lo que la omisión de ellas en la organización de las capacitaciones llevaría a resultados por debajo de lo óptimo. Por otro lado, la falta de un seguimiento hace que la labor de evaluar las capacitaciones sea más compleja. Como se vio en el PSM, la capacidad de distinguir 
entre la calidad de servicio brindado es de suma importancia para poder afirmar la efectividad de una capacitación. 


\section{CONCLUSIONES Y RECOMENDACIONES}

El análisis del mercado de capacitación microempresarial dio como resultados la identificación de las variables más importantes para explicar su demanda. Asimismo, se evaluó la efectividad de las capacitaciones en las utilidades operativas de las empresas.

Respecto a la demanda de capacitación se pudo observar que las variables más importantes para explicarla son la cantidad de equipos, educación, ciudad de origen, edad, cantidad de trabajadores y licencia municipal. Se encuentra que el perfil de un empresario que se capacita es el siguiente: Un empresario joven con estudios secundarios que labora en una empresa relativamente grande, formal, intensiva en capital y que no se encuentra en Lima.

La significancia de las variables cantidad de equipos y trabajadores muestra que las MYPE más grandes tenderían a demandar más capacitación empresarial, por lo que si se dejara la capacitación empresarial tan solo al mercado se podría incrementar la desigualdad dentro de las empresas, pues las que estén en una mejor posición demandarían más capacitación que las peores. Por tanto, estas características afectarían la efectividad del alcance de un programa de capacitación.

Por otro lado, mientras más edad tenga el empresario hay menos incentivos para capacitarse. Esto se explica en cuanto la rentabilidad de la capacitación es menor conforme se tenga más años, pues se tienen menos tiempo para usarla. Por ello, los emprendedores de edad temprana serían aquellos que reciban más capacitación y por lo tanto incrementan la probabilidad de tener éxito en el negocio.

Cabe resaltar que las variables más importantes para que el empresario se capacite son una mezcla entre las que son referidas a los mismos empresarios y a la empresa, no se encuentra ninguna prevalencia estadística de un grupo sobre el otro.

Existen distintos mecanismos en los cuales el mercado puede, a través de la capacitación empresarial, elegir que negocios triunfarán. El no ver estos incentivos puede llevar a que las políticas públicas no sean totalmente efectivas.

Al ver la política de capacitación empresarial se puede ver que no se tiene en cuenta estos temas, las capacitaciones van dirigidas en general. Es más, si las empresas dan las capacitaciones por un motivo oneroso, para poder ofrecer sus productos, se da el 
incentivo para preferir dar capacitación a las empresas que estén en buenas condiciones, pues estás tienen mayor capacidad para demandar sus servicios o productos.

Respecto a la efectividad de la capacitación, se encuentra que en promedio la mayor capacitación otorga mayores utilidades a las empresas, sin embargo, la heterogeneidad observada produce que no se pueda rechazar la hipótesis que las capacitaciones microempresariales no son efectivas en términos monetarios. Esto se debe a que en la muestra no se logra controlar por la calidad de capacitación recibida, lo cual influye de gran manera en los resultados estadísticos. Por otro lado, no se cuenta con la información acerca de la ejecución de la capacitación recibida, por lo cual se podrían tener personas que declaran haber sido capacitadas pero que nunca pusieron en práctica lo aprendido, dando menor precisión a la metodología estadística.

En las municipalidades se realizan seguimientos a las capacitaciones brindadas, un monitoreo constante y evaluación de los programas propuestos por las empresas capacitadoras. Sin embargo, en la entrevista realizada no se mencionó ningún criterio para identificar mejores programas, por ejemplo, rechazar programas que solo se dicten y no tengan ejemplos prácticos. Hace falta un detalle más completo del criterio empleado para aceptar un programa de capacitación empresarial.

Las recomendaciones del estudio surgen de manera natural a la problemática encontrada. La primera recomendación es que el foco de atención deben ser los empresarios con más necesidad de apoyo técnico, pues de no enfocarse en ellos se puede incrementar la desigualdad entre ellos. Para identificar aquellos con mayor necesidad se debe tener comunicación con los dirigentes de los centros comerciales en donde muchos de ellos operan. Una vez identificados se debe realizar un estudio de sus necesidades para luego ofrecer una capacitación apropiada que se adecue a sus demandas, asimismo, el éxito de la intervención se debe medir, entre otros indicadores, mediante el número de participantes y el porcentaje de empresarios vulnerables que asisten a la capacitación.

Asimismo, para evaluar la efectividad de las capacitaciones y la ejecución de las mismas se puede desarrollar programas de capacitación pilotos en los cuales se determine qué tipo de capacitaciones (generales, específicas, presenciales, virtuales, con material audiovisual, entre otros) tienen mejores resultados. En un primer momento se puede recolectar los datos de los empresarios durante la capacitación, luego hacer llamadas continuamente para evaluar que los usuarios están haciendo uso de lo aprendido y si es 
que las capacitaciones han tenido un resultado positivo. Luego, una vez finalizado el periodo de recolección de datos, se procedería a hacer análisis estadísticos para determinar las características del tipo de capacitaciones que fueron más exitosas en la ejecución y efectividad.

Las capacitaciones que se pretendan brindar se deben analizar cuidadosamente en cuanto a su eficacia mediante trabajos de campo. Metodológicamente, esta es la mejor opción para definir si una capacitación empresarial tiene efectos significativos sobre los resultados de la empresa, pues se tiene control de la asistencia, calidad y respuesta de los empresarios a los temas enseñados. 


\section{REFERENCIAS}

Acemoglu, D., \& Pischke, J.-S. (1999). Beyond Becker: Training in Imperfect Labour Market. The Economic Journal, 109(453), 112-142.

Agosin, M., Atal, J., Blyde, J., Busso, M., Cavallo, E., Chong, A.,... \& Zúñiga, P. (2010). La era de la productividad: Cómo transformar las economías desde sus cimientos. IDB Publications (Books).

Barron, J., Black, D., \& Lowenstein, M. (1987). Employer Size: The Implications for Search, Training, Capital Investment, Starting Wages, and Wage Growth. Journal of Labor Economics, 5(1), 76-89.

Becker, G. (1962). Investment in Human Capital: A Theorical Analysis. Journal of Political Economy, 70(5, Parte 2), 9-49.

Bloom, N., Eifert, B., Mahajan, A., McKenzie, D., \& Roberts, J. (2013). Does management matters? Evidence from India The Quarterly Journal of Economics, 128(1), 1-51.

Bloom, N., Mahajan, A., McKenzie, D., \& Roberts, J. (2010). Why Do Firms in Developing Countries Have Low Productivity? American Economic Review: Papers \& Proceedings, 100(2), 619-623.

Chiavenato, I. (1995). Introducción a la Teoría de la Administración. Caracas: Mc GrawHill.

Frazis, H., Gittleman, M., \& Joyce, M. (1998). Determinants of Training: An analysis using both employer and employee characteristics. En Key Bridge Marriott Hotel, Arlington VA, US Department of Commerce, Washington, DC.

Karlan, D., \& Valdivia, M. (2011). Teaching Entrepreneurship: Impact of Business Training on Microfinance Clients and Institutions. The Review of Economic and Statistics, 93(2), 510-527.

Instituto Nacional de Estadística e Informática (2013). Encuesta de Micro y Pequeña Empresa 2013. [Fichero de datos]. Recuperado de http://iinei.inei.gob.pe/microdatos/

Instituto Nacional de Estadística e Informática (2012). Encuesta de Micro y Pequeña Empresa 2013. [Fichero de datos]. Recuperado de http://iinei.inei.gob.pe/microdatos/

Instituto Nacional de Estadística e Informática (2011). Encuesta de Micro y Pequeña Empresa 2013. [Fichero de datos]. Recuperado de http://iinei.inei.gob.pe/microdatos/ 
La Porta, R., \& Shleifer, A. (2014). Informality and Development. Journal of Economic Perspectives, 28(3), 109-126.

Ley N. ${ }^{\circ} 28015$, Ley de promoción y formalización de la micro y pequeña empresa ( 2 de julio del 2003). Recuperado del sitio de internet del Congreso del Perú: http://www4.congreso.gob.pe/comisiones/2002/discapacidad/leyes/28015.htm

Ley N. ${ }^{\circ} 30056$, Ley que modifica diversas leyes para facilitar la inversión, impulsar el desarrollo productivo y el crecimiento empresarial (2 de julio del 2013). Recuperado del sitio de internet del Congreso del Perú: http://www2.congreso.gob.pe/sicr/cendocbib/con4_uibd.nsf/29793AB3817C627 505257EF400034156/\$FILE/30056.pdf

Loayza, N. (2008). Causas y consecuencias de la informalidad en el Perú. Revista Estudios Económicos, 15, 43-64.

Marshall, A. (1890). Principles of Economics. London: Macmillan.

Matute, G., Albújar, H., Janampa, L., Odar, R., \& Osorio, M. d. (2008). Sistema nacional de capacitación para la mype peruana. Lima: Universidad ESAN.

Ministerio de la Producción. (2017). Las mipyme en cifras 2015. Lima: Ministerio de la Producción.

Monge-González, R., Rodríguez-Álvarez, J. A., \& Torrentes-García, L. (2013). El impacto de la capacitación sobre la adopción de mejores prácticas administrativas y el desempeño de las MiPyME en Costa Rica (No. IDB-WP442). IDB Working Paper Series.

O'Connell, P., \& Byrne, D. (2012). The Determinants and Effects of Training at Work: Bringing the Workplace Back In. European Sociological Review, 28(3), 283300 .

Oosterbeck, H. (1998). Unravelling supply and demand factors in work-related training. Oxford Economic Papers, 50(2), 266-283.

Palacios, C. (2013). Determinantes de la capacitación en Chile (tesis de titulación). Universidad de Chile, Santiago, Chile. Recuperada de http://repositorio.uchile.cl/bitstream/handle/2250/113967/Tesis\%20Palacios\%20 Hucke.pdf? sequence $=1$

Villarán, F. (2010). Políticas e instituciones de apoyo a la micro y pqeuña empresa (MYPE) en el Perú. En C. Ferraro, \& G. Stumpo, Políticas de apoyo a las pymes en América Latina (págs. 343-386). Santiago de Chile: Naciones Unidas.

Walker, F. (1887). The Source of Business Profits. The Quarterly Journal of Economics, 1(3), 265-288.

Ypeij, A. (2006). Produciendo contra la pobreza. Lima: Instituto de Estudios Peruanos. 


\section{BIBLIOGRAFÍA}

Bertrand, M., Mullainathan, S., \& Shafir, E. (2004). A Behavioral-Economics View of Poverty. American Economic Review, 94(2), 419-423.

Brañas, P. (2011). Economía experimental y del comportamiento. Barcelona: Antoni Bosch.

Darley, J., \& Batson, D. (1973). From Jerusalem to Jericho: A Study of Situational and Dispositional Variables in Helping Behavior. Journal of Personality and Social Psychology, 27(1), 100-108.

Hosmer, D., \& Lemeshow, S. (2000). Introduction to the logistic regression model. Applied Logistic Regression. Nueva York: John Wiley \& Sons, Inc.

Kahneman, D., \& Tversky, A. (1984). Choices, Values, and Frames. American Psychologist, 39(4),341-350. 